\title{
Heat shock stimulation of a tilapia heat shock protein 70 promoter is mediated by a distal element
}

\author{
Alfredo MOLINA, Emmanuel DI MARTINO, Joseph A. MARTIAL and Marc MULLER ${ }^{1}$ \\ Laboratoire de Biologie Moléculaire et Génie Génétique, Université de Liège, Institut de Chimie B6, B-4000 Sart-Tilman, Belgium
}

We reported previously that a tilapia (Oreochromis mossambicus) heat shock protein 70 (HSP70) promoter is able to confer heat shock response on a reporter gene after transient expression both in cell culture and in microinjected zebrafish embryos. Here we present the first functional analysis of a fish HSP70 promoter, the tiHSP70 promoter. Using transient expression experiments in carp EPC (epithelioma papulosum cyprini) cells and in microinjected zebrafish embryos, we show that a distal heat shock response element (HSE1) at approx. -800 is predominantly responsible for the heat shock response of the tiHSP70 promoter. This element specifically binds an inducible transcription factor, most probably heat shock factor, and a constitutive factor. The constitutive complex is not observed with the non-functional, proximal HSE3 sequence, suggesting that both factors are required for the heat shock response mediated by HSE1.

Key words: HSP70, transcription, zebrafish.

\section{INTRODUCTION}

The first description in Drosophila melanogaster of a subset of cellular proteins that are induced on heat shock (HSP proteins) [1] has triggered a remarkable amount of research on these proteins in various organisms and on their function in stress tolerance. This heat shock response is found universally from bacteria to humans and the HSP genes are evolutionarily among the most conserved genes.

The most important and most studied heat shock proteins form the heat shock protein 70 (HSP70) family. They have essential roles in protein metabolism under normal and stress conditions, including de novo protein folding, membrane translocation, degradation of misfolded proteins and other regulatory processes. Although their binding to an unfolded polypeptide chain results in the stabilization of this state, their controlled release might allow progression along the folding pathway [2]. Because of their functions, HSP70s have been included in the large family of chaperones. Their expression is regulated by environmental and physiological stress and non-stressful conditions such as cell growth and development [3].

The regulation of hsp70 gene expression occurs mainly at the transcriptional level. Analysis of HSP genes, mainly from Drosophila, and comparison of different heat shock regulatory regions led to the identification of a palindromic 'heat shock element' (HSE), CNNGAANNTTCNNG [4-6]. More recent results suggested that HSEs are composed of contiguous arrays of a variable number of the highly conserved sequence NGAAN arranged in alternating orientation [7-9]. These elements bind the trans-acting heat shock factors (HSFs), which are normally present in the cytoplasm in a monomeric form (with no DNAbinding activity) and which, on heat stress, form trimers and migrate to the nucleus to bind HSEs with high affinity [10-15]. In addition to the HSEs, the basal and induced transcription of hsp70 genes require the presence of binding sites, such as GC, CAAT and GAGA boxes, for other specific transcription factors [16-18].
In fish, cDNA species coding for HSP70 (for example from rainbow trout [19], medaka [20] or zebrafish [21,22]) have been described and a heat-shock-induced increase in mRNA levels has been shown. Genomic sequences of an HSP70 gene family have been reported in the teleost Fugu rubripes [23]. The 5'flanking sequences of the HSP70-coding regions were described for zebrafish [24] and tilapia [25] and these promoters were shown to mediate the heat shock response. However, no information on the sequences involved in transcriptional regulation of fish HSP70 genes has been available until now.

We recently reported the isolation and characterization of the Mozambique tilapia (Oreochromis mossambicus) gene encoding HSP70, including approx. $1 \mathrm{~kb}$ of regulatory sequences [25]. We showed that the tilapia HSP70 promoter is able to confer ubiquitous and heat-shock-controlled expression on a reporter gene, both in fish cells and in microinjected zebrafish embryos. In the present study we focused on the specific sequences controlling the stress response of the tilapia HSP70 promoter.

\section{MATERIALS AND METHODS}

\section{Oligonucleotides}

All of the oligonucleotides shown in Table 1 were obtained from Eurogentec (Seraing, Belgium).

\section{Plasmid constructs}

The following plasmids have been described previously: pGCV-Lac-Z [26], pGCV(-SV) [25], tiHSP70-1.0LacZ [25] and ptkLuc [27] (in which tk stands for thymidine kinase). pCMVsGFPLuc was kindly provided by R. Day (Health Sciences Center, University of Virginia, Charlottesville, VA, U.S.A.) [28]. The p0GL reporter plasmid, containing a cDNA coding for a fusion protein of green fluorescent protein (GFP) and luciferase (Luc) was obtained by transferring the reporter gene GFP-Luc from the pCMVsGFPLuc vector digested by the restriction enzymes $X h o I$ and $X b a I$ and then inserted into the vector pGCV $(\mathrm{SV}-)$.

Abbreviations used: EMSA, electrophoretic mobility-shift assay; EPC, epithelioma papulosum cyprini; GFP, green fluorescent protein; HSE, heat shock element; HSF, heat shock factor; HSP70, heat shock protein 70; Luc, luciferase; tiHSP70, tilapia HSP70; TK or tk, thymidine kinase.

1 To whom correspondence should be addressed (e-mail m.muller@ulg.ac.be). 
Table 1 Oligonucleotides used in this study

\begin{tabular}{|c|c|}
\hline Oligonucleotide & Sequence \\
\hline \multicolumn{2}{|c|}{$5^{\prime}$-deletion constructs } \\
\hline GC1DEL & 5'-GAGCTCGAGAAGAGAAGAACAAGAGC-3' \\
\hline TTCDEL & 5'-GAGCTCGATCCTCATCTGTCACCGAG-3' \\
\hline GAADEL & 5'-GAGCTCATAAAATGTTCAGGGAC-3' \\
\hline XBOX1DEL & 5'-GAGCTCCCATTTTAATCATTAATGAAATC--3' \\
\hline BOX2DELX & 5'-GAGCTCATCTTGAAAATTCAGTATTAATCGTGC-3' \\
\hline HSE2DEL & 5'-GAGCTCTAATCATTAATGGCTGGATG-3' \\
\hline XBOX3DEL & 5'-GAGCTCATGGCTGGATGAAAACTCAG-3' \\
\hline HINDIII & 5'-CAAAGCTTTATTTTAAAAAAAGAAGAAC-3' \\
\hline \multicolumn{2}{|c|}{ HSP-TK hybrid and HSP70 promoter internal deletions } \\
\hline BLUESCF & 5'-AAGCTTGCGGCCGCCACCGCGGTGGAGCTC-3' \\
\hline HSE1TK & 5'-TCTAGAGGATCCTAATAAATTAATGATTAAATAAAC-3' \\
\hline \multicolumn{2}{|c|}{ HSP70 mutant promoter constructs (only coding-strand primers) } \\
\hline CAAT1MUT & 5'-GAGCACTCTGATTCGATGAGAGTCCCG-3' \\
\hline HSE1MUT1 & $5^{\prime}$-GAGAGTCCCGGCAAAGCTCGAGAACTC-3' \\
\hline HSE1MUT2 & $5^{\prime}$-GGTAAAGCTCGÄTAACTCACGAGAAGG-3' \\
\hline GCMUT & 5'-CTḠGAAGAGACĀAACGGGGGAGAAGAGAAGAAC-3' \\
\hline XBOX1MUT & 5'-CATTTTTAAAGTTTATTTĀATCG \\
\hline XBOX2MUT & 5'-ATTAACCATTTTAATCG TTAATḠAAATCTTGAAAATTCAG-3' \\
\hline HSE2MUT & 5'-TAATCATTAATGAAATC̄TTTAAAATTCAGTATTAATCGTGCAGAG-3' \\
\hline ASOC1MUT & 5'-CTTGAAAATTCAGTATTAC̄TCGTGCATAGTCACGATTAAATTTA-3' \\
\hline XBOX3MUT & 5'-CACGATTAAATTTATTTAATCGTTAATGGCTGGATGAAAACTC-3' \\
\hline CAAT2MUT & 5'-ATATCTAAAGGAACCCGAATTATAAAACTGCATG-3' \\
\hline HSE3MUT & 5'-CATGGCTGTCTTCTATÄAAATCAGTATTAATCG-3' \\
\hline ASOC2MUT & 5'-GAAAATTCAGTATTAC TCGTGCATAGTCATGAGACTATATTC-3' \\
\hline \multicolumn{2}{|l|}{ EMSA } \\
\hline HSE1 & 5'-GGGAAAGCTCGAGAACTCACGAGAAGG-3' \\
\hline HSE1MUT2 & 5'-GGTAAAGCTCGATAACTCACGAGAAGG-3' \\
\hline HSE2 & 5'-GAAATCTTGAAAATTCAGTAT-3' \\
\hline HSE2MUT & 5'-GAAATCTTITAAAATTCAGTAT-3' \\
\hline HSE3 & 5'-GTCTTCTAG̈AAAATTCAGTAT-3' \\
\hline HSE3MUT & $5^{\prime}$-GTCTTCTAGAAAATTCAGTAT-3' \\
\hline Xbox2 & 5'-GTTTATTTAATCATTAATTTATTAAC-3' \\
\hline XBOX2MUT & 5'-GTTTATTTAATCGTTAATTTATTAAC-3' \\
\hline
\end{tabular}

Plasmid pGCV(SV-) was digested with HindIII and SacI, filled in with the Klenow enzyme and religated to destroy both sites. This plasmid was digested by NotI and used to insert a NotI cassette containing the HSP70 entire promoter from the tiHSP70$1.0 \mathrm{LacZ}$ vector. This vector (tiHSP70LacZ2) was used to construct the deletion mutants. PCR fragments were obtained with tiHSP70-1.0LacZ as template with the following primer pairs: GC1DEL, TTCDEL, GHSE1DEL, XBOX1DEL, XBOX2DEL, HSE2DEL, XBOX3DEL respectively as $5^{\prime}$ primers and HINDIII as 3 ' primer, or BLUESCF with HSE1TK. All fragments were directly cloned into pCR ${ }^{\circledR} \mathrm{II}$ from Invitrogen (Carlsbad, CA, U.S.A.) for sequencing. The fragments were excised by digestion with $S a c I$ and HindIII and used to replace the SacI/HindIII fragment ( $5^{\prime}$ end and -401 of the tiHSP70 promoter) in tiHSP70LacZ2 to obtain the constructs pGC1DEL, pTTCDEL, pGAADEL, pXBOX1DEL, pXBOX2DEL, pHSE2DEL and PXBOX3DEL. Construct pHSE1DEL was obtained by SacI/XhoI digestion of tiHSP70LacZ2, Klenow fill-in and religation. Construct pHSE3DEL was obtained by $\mathrm{SacI} / \mathrm{XbaI}$ digestion of tiHSP70LacZ2, Klenow fill-in and religation. The internal promoter deletion construct $\mathrm{pHSEDis}$ was obtained by inserting the BLUESCF-HSE1TK SacI/XbaI fragment $(-821 / 524)$ into tiHSP70LacZ2 previously digested with SacI and $\mathrm{Xba \textrm {I }}(-182)$.

The deleted promoter fragments were excised with Not I and cloned into the NotI site of the promoterless p0GL vector to obtain the tiHSP70 promoter deletion reporter constructs pHSE1DELGL (-806), pGC1DELGL (-704), pTTCDELGL
(-667), pGAADELGL (-574), pXBOX1DELGL (-523), pXBOX2DELGL (-503), pHSE2DELGL (-455), pXBOX3DELGL (-446), pHSE3DELGL $(-182)$ and pHSEDisGL. Similarly, the wild-type promoter was excised from the tiHSP70-1.0LacZ by NotI and inserted into p0GL to obtain the tiHSP70-1.0GL.

The thymidine kinase (TK)-HSP hybrid promoter construct pHSEDistkLuc was obtained by inserting the PCR fragment BLUESCF-HSE1TK into the ptkLuc vector via the HindIII and BamHI sites.

\section{Site-directed mutagenesis}

Site-directed mutagenesis of the wild-type promoter construct tiHSP70-1.0GL was performed with the QuikChange ${ }^{\text {(im }}$ SiteDirected Mutagenesis kit (Stratagene, La Jolla, CA, U.S.A.) in accordance with the manufacturer's instructions. In brief, the target plasmid was amplified by PCR with oligonucleotides containing the desired mutation and a high-fidelity polymerase. After that, the methylated template DNA was digested with $D p n I$ and the remaining mutated DNA was transformed into a supercompetent Escherichia coli. The oligonucleotides used were as described above.

All mutations were verified by sequencing. The constructs obtained were pCAAT1MUT, pHSE1MUT1, pHSE1MUT2, pGCMUT, pXBOX1MUT, pXBOX2MUT, pHSE2MUT, pASOC1MUT, pXBOX3MUT, pCAAT2MUT, pHSE3MUT and pASOC2MUT. 


\section{Cell culture and transfection experiments}

EPC (epithelioma papulosum cyprini) cells, derived from carp epidermal herpes-virus-induced hyperplasia lesions [29], were grown in BHK-21 medium supplemented with $10 \%$ (v/v) fetal bovine serum and $1 \%(\mathrm{w} / \mathrm{v})$ penicillin/streptomycin at $24{ }^{\circ} \mathrm{C}$ in air $/ \mathrm{CO}_{2}$ (19:1). Purified plasmid DNA (20 pmol) was transfected in $800 \mu \mathrm{l}$ of resuspended cells $\left(1.5 \times 10^{6}\right.$ cells $/ \mathrm{ml}$ of medium $)$ by electroporation in $4 \mathrm{~mm}$ cuvettes with a single pulse $(250 \mathrm{~V}$, $1500 \mu \mathrm{F}$ ) delivered by an Easyject apparatus (Eurogentec, Seraing, Belgium). Transfected cells were immediately transferred to four culture dishes $(55 \mathrm{~mm})$. After $48 \mathrm{~h}$ the cells were harvested by scraping, washed twice in PBS and resuspended in lysis buffer [30]. Protein concentration and LacZ activity were determined as described in [31].

The mutant HSP70 promoter constructs were transfected with the LIPOFECTAMINE Plus reagent from Gibco BRL (Gaithersburg, MD, U.S.A.) by using 1 pmol of plasmid per well in 24-well plates containing $1.0 \times 10^{5}$ cells per well.

Heat shocks were performed by transferring the plates previously sealed with Parafilm in a $37^{\circ} \mathrm{C}$ thermoregulated bath. After treatment, the cells were returned for $6 \mathrm{~h}$ at $24^{\circ} \mathrm{C} . \mathrm{Cd}^{2+}$ treatment was performed by changing the medium $24 \mathrm{~h}$ after transfection to medium supplemented with $\mathrm{CdCl}_{2} ; 12 \mathrm{~h}$ later the cells were harvested and the luciferase activity was determined in a multilabel counter (Wallac Victor ${ }^{2}$; PerkinElmer Life Sciences, Norwalk, CT, U.S.A.).

\section{EMSA}

Cell nuclear extracts were prepared from control cells $\left(24^{\circ} \mathrm{C}\right)$ and cells were heat shocked $\left(37^{\circ} \mathrm{C}\right)$ for $1 \mathrm{~h}$. The cells were immediately harvested and incubated for $15 \mathrm{~min}$ in hypotonic buffer [10 mM Hepes ( $\mathrm{pH} 7.8) / 10 \mathrm{mM} \mathrm{KCl} / 0.1 \mathrm{mM}$ EDTA/ $0.1 \mathrm{mM}$ EGTA/1 mM dithiothreitol/0.5 mM PMSF] before the addition of $25 \mu \mathrm{l}$ of $10 \%$ (v/v) Nonidet P40. After centrifugation for $30 \mathrm{~s}$ at $12000 \mathrm{~g}$, the nuclear pellet was resuspended in binding buffer [20 mM Hepes (pH 7.8)/400 mM KCl/20\% (v/v) glycerol $/ 2 \mathrm{mM}$ dithiothreitol] containing an anti-protease mixture (Boehringer Mannheim, Indianapolis, IN, U.S.A.), then frozen in liquid nitrogen, thawed on ice and centrifuged for $30 \mathrm{~min}$ at $12000 \mathrm{~g}$. The supernatant was stored at $-70^{\circ} \mathrm{C}$ and the protein concentration was determined by using a Bradford assay [32] with BSA as a standard.

Double-stranded oligonucleotides were labelled by using the T4 polynucleotide kinase (Pharmacia, Piscataway, NJ, U.S.A.) with $\left[\gamma^{32} \mathrm{P}\right]$ ATP.

Gel retardation analysis was performed with $2 \mu \mathrm{g}$ of nuclear protein extract, $1 \mu \mathrm{g}$ of poly(dI:dC) and 30000 c.p.m. of ${ }^{32} \mathrm{P}-$ labelled DNA fragments, in $5 \mathrm{mM}$ Hepes $(\mathrm{pH} 7.8) / 100 \mathrm{mM}$ $\mathrm{KCl} / 5 \%$ (v/v) glycerol. The resulting DNA-protein complexes were resolved by electrophoresis on a pre-run $5 \%(\mathrm{w} / \mathrm{v})$ native polyacrylamide gel, with $0.5 \times$ Tris/borate/EDTA as running buffer. The gel was dried, exposed to an X-ray-sensitive Imaging screen-K (Bio-Rad, Hercules, CA, U.S.A.) and the image was analysed on a Personal Molecular Imager FX System (Bio-Rad).

\section{Microinjection procedure}

Fish care and embryo rearing were performed as described [33]. Eggs were collected and microinjected $(300 \mathrm{pl}, 50 \mathrm{ng} / \mu \mathrm{l})$ at the one-cell stage, targeting to the cytoplasm/yolk boundary of the zygote. After microinjection the embryos were incubated at $28.5^{\circ} \mathrm{C}$ in small tanks containing $500 \mathrm{ml}$ of Holtfreter's solution to permit correct development. Heat shock treatment was performed on 1-day-old embryos at $37^{\circ} \mathrm{C}$ for $1 \mathrm{~h}$, followed by recovery for $2 \mathrm{~h}$ at $28.5^{\circ} \mathrm{C}$.

\section{Fluorescence microscopy}

The GFP-Luc fusion protein was revealed in the embryos by using an Olympus BX60 microscope equipped with a ColorView8 device camera and analySIS ${ }^{\circledR} 3.0$ imaging software (Soft Imaging System, Münster, Germany).

\section{RESULTS}

\section{Mutation of the tiHSP70 promoter}

As a first approach to the identification of the sequences involved in the transcriptional regulation of the tiHSP70 promoter, transient expression studies were performed in carp EPC cells. Putative transcription-factor-binding sites had been identified previously [25], as well as a sequence (Xbox) that is repeated three times in the tiHSP70 promoter and for which no putative binding factor could be defined (see Figure 1A). Reporter constructs containing the GFP-Luc (GL) gene driven by either the entire regulatory region (tiHSP70-1.0GL) or successive $5^{\prime}$ deletions therein were tested. The end points of the deletions were chosen such that each deleted an additional HSE, Xbox, GC box or other interesting feature (TTC or AAG; see Figure 1A). Heat shock for $1 \mathrm{~h}$ at $37^{\circ} \mathrm{C}$ resulted in a 600 -fold induction of the largest promoter construct tiHSP70-1.0GL, as expected (Figure 1B). Deletion of the HSE1 sequence (and an inverted CAAT box; see below) did not affect the basal level activity but almost completely abolished the heat shock response of the tiHSP70 promoter. The 10-fold residual stimulation was further decreased (2-fold) on deletion of the GC box. Successive further deletions down to -182 did not affect basal level activity; only a non-significant heat shock (2-3-fold) response was observed.

A
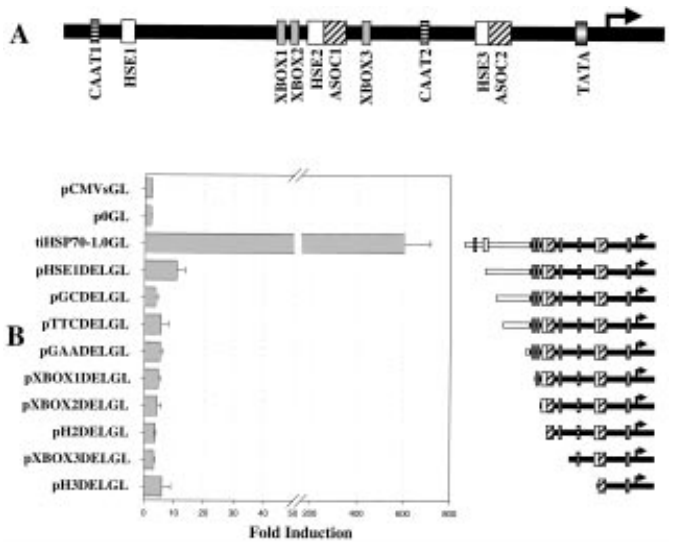

Figure 1 Deletion analysis of the tiHSP70 promoter by transient expression experiments

(A) Schematic representation of the tiHSP70 promoter. TiHSP70-1.0GL contains the complete isolated tiHSP70 control region $(-851 /+157)$, which was fused upstream from the ATG codon of the GFP-Luc (GL) reporter gene. The putative transcription-factor-binding sites are indicated and the end points of the $5^{\prime}$ deletion mutants are shown. (B) Transient expression of tiHSP70 promoter $5^{\prime}$ deletion constructs in carp cultured EPC cells. EPC cells transfected with one of the tiHSP70 reporter constructs and pGCV-LacZ (internal transfection control) were subjected or not to a heat shock for $60 \mathrm{~min}$ at $37^{\circ} \mathrm{C}$. The cells were left for $2 \mathrm{~h}$ at $28^{\circ} \mathrm{C}$ for recovery, then harvested; both lacZ and luciferase activities were determined. Luciferase activity was normalized to the control lacZ activity. Results are shown as fold inductions relative to nontreated cells (means \pm S.D. for triplicate experiments performed at least twice). 


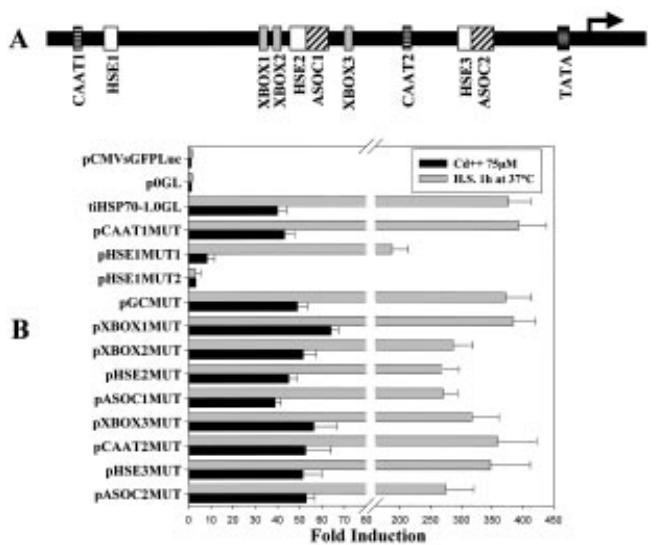

Figure 2 Transient expression of site-specific mutant tiHSP70 promoter constructs in carp cultured EPC cells

The mutated sites are shown in (A) and indicated in the names of the constructs in (B). Results are shown as fold inductions after heat shock and treatment with $75 \mu \mathrm{M} \mathrm{Cd} \mathrm{d}^{2+}$ relative to nontreated cells (means \pm S.D. for quadruplicate experiments performed at least three times).

To assess more directly the role of each of the individual putative binding sites identified by computational analysis, point mutations were generated in the tiHSP70-1.0GL promoter construct for all of these sites (HSE1, HSE2, HSE3, Xbox1, Xbox2, Xbox3, two CAAT boxes and one GC box). Two additional repeat sequences (ASOC1 and ASOC2) were also mutated. All of these constructs were tested for their ability to respond to heat shock and to treatment with heavy-metal ions $\mathrm{Cd}^{2+}$. None of the mutations seemed to affect the basal level activity (results not shown). Mutation of the upstream CAAT box (pCAAT1MUTGL) did not affect the stimulation by heat (Figure 2). A single point mutation in HSE1 decreased the heat shock response by approx. 2-fold (from 376-fold to 187-fold) but significantly decreased the stimulation by $\mathrm{Cd}^{2+}$. Close inspection of the HSE1 sequence revealed the presence of several NGAAN sequences in this region. When two of these elements were mutated, in pHSE1MUT2GL, both responses were completely abolished. Mutations in any of the other sites hardly affected the response to either heat shock or heavy metal. In particular, no indication was observed for a possible contribution of the two more proximal HSE elements, HSE2 and HSE3, which would have been detected in the presence of an intact HSE1.

Taken together, these results indicate that the most proximal tiHSP70 promoter region $(-182)$ is sufficient for its basal activity and that HSE1 is absolutely required for the heat shock response of the tiHSP70 promoter.

\section{Independent distal heat shock response element}

The predominant role of the distal HSE1 element as revealed by mutational analysis prompted us to study this element in more detail. When the region $-851 /-524$, containing HSE1, was inserted upstream of the minimal tiHSP70 promoter, thereby deleting the central tiHSP70 promoter portion including HSE2 and HSE3, the heat shock response was similar (836-fold) to that of the complete promoter (547-fold; Figure 3). In addition, insertion of the same region upstream of the heterologous herpes simplex TK promoter was sufficient to confer heat shock inducibility on the constitutive TK promoter (Figure 3). These results indicate that the distal HSE1 element is able to mediate a transferable heat shock response.

\section{Transient expression in zebrafish embryos}

The transcriptional activities of the wild-type tiHSP70 promoter and its double mutant in HSE1 (pHSE1MUT2GL) were also compared in whole animals by microinjection into zebrafish eggs (Figure 4C). Injection of the wild-type promoter (tiHSP70$1.0 \mathrm{GL}$ ) (Figures 4A and 4B) resulted in strong expression only after heat shock ( 0 of 43 positive individuals without heat shock, compared with 14 of 45 after heat shock). As described previously [25], no preference for a specific tissue was observed. In contrast, when the pHSE1MUT2GL construct was injected, no signal was observed even when the embryos were submitted to heat shock ( 0 of 56 and 0 of 65 individuals with and without heat shock respectively). As expected, the positive control (pCMVsGFPLuc) directed constitutive expression (24 of 73 positives with heat treatment; 31 of 68 positives without heat treatment) (results not shown).

\section{EMSA}

To examine specific heat-shock-induced DNA-binding activities in the tiHSP70 promoter, we performed EMSAs with nuclear extract from heat shocked or untreated EPC cells. We focused on

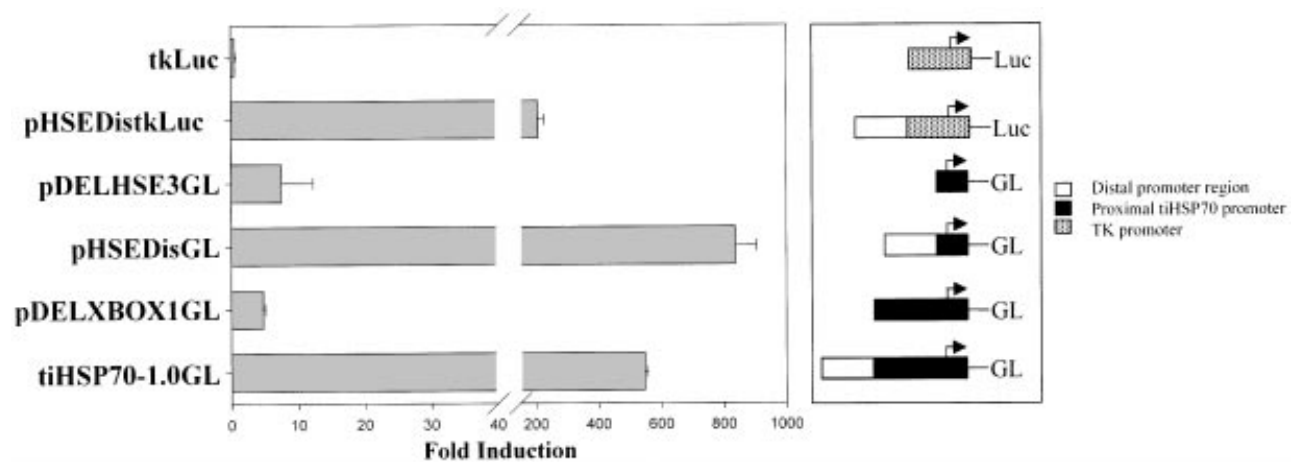

Figure 3 Heat shock response mediated by the distal HSE1 region

The tiHSP70 distal promoter fragment $-851 /-524$ was fused upstream of the minimal tiHSP70 promoter or the heterologous TK promoter. The transcriptional activity was compared in treated and untreated cells with that of the corresponding constructs lacking this fragment. The activities of the complete tiHSP70 promoter (tiHSP70-1.0GL) and the promoter deleted of the distal HSE1 region (pDELXBOX1GL) are shown as controls. 


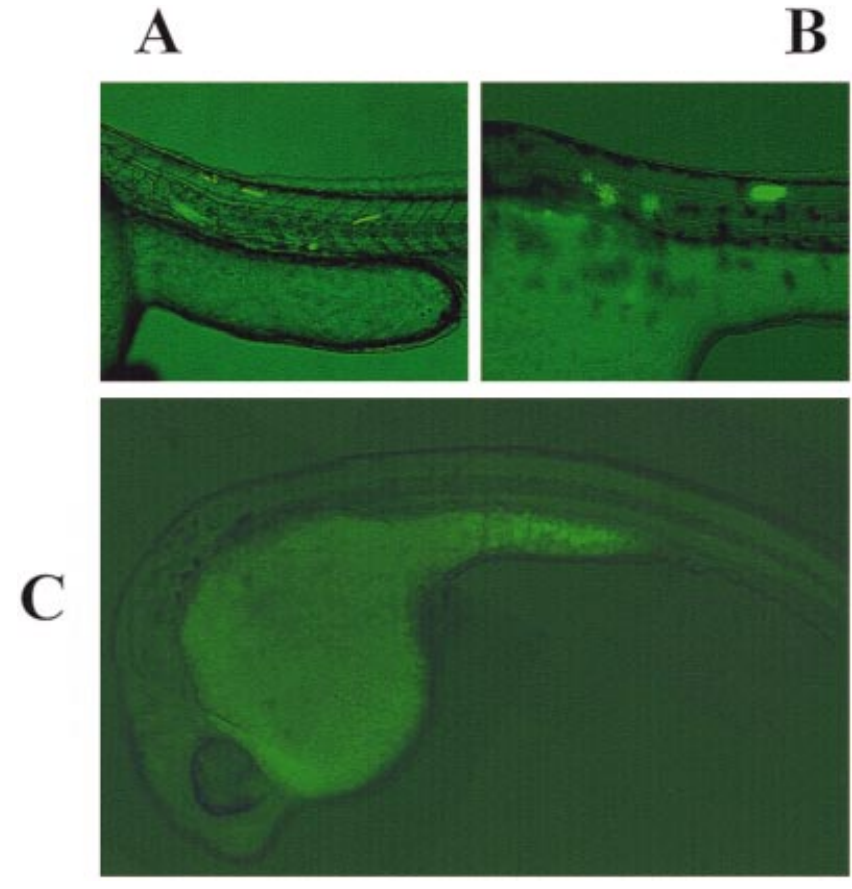

Figure 4 Transient expression of tilapia HSP70 promoter constructs in zebrafish embryos

(A-C) Zebrafish embryos were microinjected at the one-cell stage with the tiHSP70-1.0GL (two different positive individuals are shown; $\mathbf{A}$ and B) and the mutated pHSE1MUT2GL (C) linearized constructs. Embryos 1 day old were submitted to a heat shock at $37^{\circ} \mathrm{C}$ for $1 \mathrm{~h}$ and then left to recover for $2 \mathrm{~h}$ at $28.5^{\circ} \mathrm{C}$; the fluorescence due to expression of the GFP-Luc fusion protein was analysed by microscopy.
A

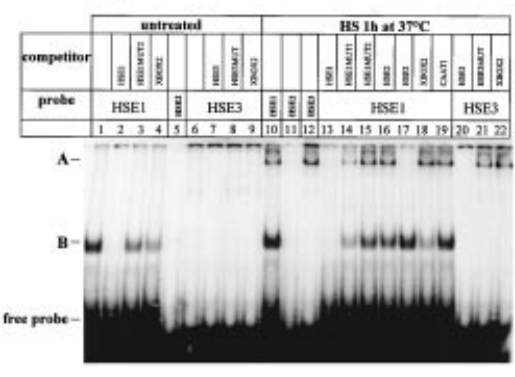

B

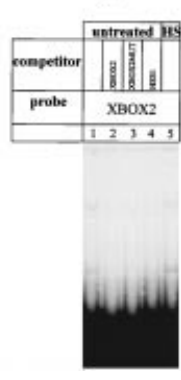

Figure 5 Transcription factors binding to the tiHSP70 promoter

EMSA experiments were performed with (A) the HSE1, HSE2 and HSE3 probes and (B) the XBOX2 probe with nuclear extracts from control EPC cells and cells subjected to a heat shock for $1 \mathrm{~h}$ at $37^{\circ} \mathrm{C}$. The inducible and constitutive complexes, $A$ and $B$ respectively, are indicated at the left of the gel. Competition experiments with a 200 -fold excess of the indicated unlabelled oligonucleotides are shown.

the HSE1, HSE2 and HSE3 sequences (oligonucleotides HSE1, HSE2 and HSE3) because they contain putative binding sites for the heat-shock-specific transcription factor HSF.

A slowly migrating DNA-protein complex (A) was formed on the HSE1 and the HSE3 probes in the heat-shock-treated extract (Figure 5A, lanes 10 and 12) that was absent from the control extract (lanes 1 and 6). The complex, representing a heat-shockinduced DNA-binding activity, was not observed when the HSE2 probe was used (Figure 5A, lane 11). An additional faster- migrating complex (B) appeared only on HSE1 in nuclear extracts from both control and treated cells (Figure 5A, lanes 1 and 11), suggesting that its appearance corresponded to the binding of a constitutive factor. This complex was not observed on HSE2 or HSE3 (Figure 5A, lanes 11 and 12), indicating that its formation was specific for HSE1.

Competition experiments were performed with extracts from heat shocked and normal cells to determine the specificity of the $\mathrm{A}$ and $\mathrm{B}$ complexes. The disappearance of both complexes on HSE1 was observed on the addition of a 200 -fold molar excess of unlabelled HSE1 oligonucleotide (Figure 5A, lanes 2 and 13) and the single mutated HSE1MUT1 seemed to compete slightly for both complexes (lanes 3 and 14), whereas no competition was observed with the doubly mutated HSE1MUT2 or the unrelated CAAT1 box (lanes 15 and 18). Only complex A was displaced by an excess of unlabelled HSE3 but not by the HSE2 oligonucleotide (Figure 5A, lanes 16 and 17). The unrelated XBOX2 did not compete for the heat-shock-specific A complex formed on HSE1 but surprisingly it seemed to compete for the constitutive complex, B (Figure 5A, lanes 4 and 18).

Complex A formed on the HSE3 was competed for by HSE3, but not by the HSE3MUT or the XBOX2 oligonucleotides, indicating that the complex was specific.

When the XBOX2 probe was used, only very weak shifted bands were observed with both untreated and treated extracts (Figure 5B, lanes 1 and 5), none of which corresponded to complex B. These complexes were competed for by XBOX2, XBOX2MUT and HSE1 oligonucleotides, suggesting that they were non-specific.

In conclusion, a heat-shock-inducible complex, presumably the trimeric HSF, binds specifically to the HSE1 and HSE3 elements and an additional specific constitutive binding activity is observed on HSE1 that is weakly competed for by XBOX2.

\section{DISCUSSION}

We previously reported the cloning of the tiHSP70 promoter and showed that it is able to confer heat shock response on a reporter gene by transient expression both in cell culture and in microinjected zebrafish embryos [25]. Computer analysis of the regulatory region revealed no obvious similarities to other HSP70 promoters or $5^{\prime}$ leader sequences. However, three putative HSEs were detected, as were several potential binding sites for ubiquitous transcription factors (TATA, CAAT and GCGGG boxes). Here we have analysed in detail the contribution of individual elements to the transcriptional activity of the tiHSP70 promoter and investigated the presence of transcription factors involved in heat shock response in carp EPC cells.

Basal level expression driven by the tiHSP70 promoter was not affected by any of the $5^{\prime}$ deletion or point mutations tested here, suggesting that the most proximal promoter region, downstream of -182 , is sufficient for expression. This region contains the TATA box at -38 [25] and a non-consensus CAAT box (tcaat), which probably explain the basal, low activity.

Several observations indicate that the distal HSE1 element centred on -800 is mainly responsible for the heat shock response of the tiHSP70 promoter: (1) deletion of the region $-851 /-806$ resulted in a nearly complete loss of induction; (2) the HSE1 element binds a specific, heat-shock-inducible factor in EMSA experiments; (3) mutation of HSE1 at two positions (pHSE1MUT2GL) abolished induction by heat shock, whereas mutation of the distal CAAT box (CAAT1), also present in this region, had no effect; and (4) the same mutation in HSE1 abolished the stimulation in microinjected zebrafish embryos. The single point mutation in pHSE1MUT1GL probably 


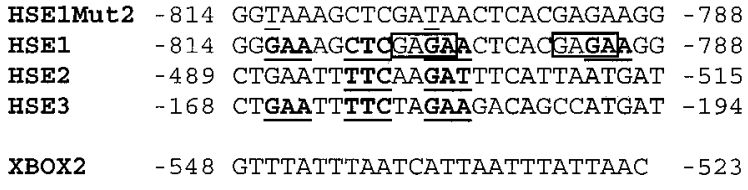

Figure 6 Sequence comparison of the functional HSE1 element with the inactive HSE1MUT2, HSE2, HSE3 and XBOX2 regions

Identical bases in the HSE sequences are shadowed, the mutations in HSE1MUT2 are underlined, the GAA motifs are in bold and underlined, and the GAGA sequences are boxed.

decreases the binding affinity of HSE1, thereby affecting the weak stimulation by $\mathrm{Cd}^{2+}$ and, to a smaller extent, the much stronger heat shock effect. Mutation of any of the other putative binding sites did not alter the stress stimulation, strongly suggesting that HSE1 alone is responsible for the effect. Accordingly, region $-851 /-524$, containing only HSE1, was able to mediate induction by heat shock when fused upstream of the minimal tiHSP70 or the heterologous TK promoter. Taken together, these observations show that the other putative binding sites, most importantly the more proximal HSE2 and HSE3, are not involved in heat shock response. This is in striking contrast with other previously described HSP70 promoters, where the stimulation is mediated by the most proximal 150-200 bp region and functional HSEs are often located very close to the transcription start site [17,34-36].

DNA binding assays reveal that HSE1 is able to form two different, specific complexes with nuclear extracts from carp EPC cells. One complex, B, is observed with both treated and untreated cells, whereas the other, A, is obtained only after heat shock and probably contains the carp HSF. Consistent with the functional analysis was the failure to detect protein interaction detected with the HSE2 probe. However, HSE3 surprisingly gave rise to the same inducible complex as HSE1 and was able to compete for HSE1 complex A.

The fact that complex $\mathrm{B}$ is not formed with probe HSE3 and is not competed for by an excess of unlabelled HSE3 suggests that the exclusive functionality of HSE1 is caused by the formation of this constitutive complex. The lack of function of HSE3 despite the presence of an HSF could be due to the absence of this constitutive factor. Interestingly, the singly mutated HSE1MUT1 weakly competes for both complexes on HSE1, whereas XBOX2 competed, also weakly, for the constitutive B complex. This weak competition probably reflects a low affinity, as no similar complex is observed when XBOX2 is used as a probe. Constitutive binding to functional HSEs has been described before in extracts from Xenopus oocytes [37] and from rat (Rat-1) [38] and human (HeLa) cells [39]. The mammalian complex was shown to be identical with the $\mathrm{Ku}$ autoantigen, a DNA-dependent protein kinase involved in the repair of doublestrand breaks in DNA [40] and the protection of telomeres [41]. The HSF1 and $\mathrm{Ku}$ sites overlap [14] and both factors mutually compete for binding to a human HSE site. $\mathrm{Ku}$ binding decreases after heat shock, when HSF binding increases, and reappears after the recovery phase [14]. In carp EPC cells, no indication of a decreased formation of constitutive complex $\mathrm{B}$ or a binding competition with HSF was observed. In contrast, our results suggest that the formation of complex B is correlated with the functionality of HSE1.

When the sequences of the three putative HSE elements are compared, HSE1 seems to be characterized by the presence of three correctly spaced NGAAN motifs and one inverted, im- perfect NTTCN motif. HSE3 contains three correctly spaced motifs, explaining the observed HSF binding, whereas HSE2 presents only two correct NGAAN sites (Figure 6). No obvious sequence similarity was detected between $\mathrm{XBOX} 2$ and the HSE elements. Close inspection of the HSE1 sequence revealed the presence of two GAGA sequences that are absent from the other elements. The mutation in HSE1MUT2 eliminates one of these sites (Figure 6). GAGA-box-binding factor ('GAF') was previously shown to be involved in the heat shock response of HSP70 genes in Drosophila [42] and Xenopus [43]. This factor binds its specific sites constitutively and is therefore another candidate for the constitutive complex formed on HSE1. No definitive answer can yet be given about the identity of this factor. It remains to be determined whether this distal HSE1 element is a special feature of fish HSP70 genes.

In conclusion, we show that the distal HSE1 element is responsible for the stimulation of the tiHSP70 promoter by cellular stress in carp EPC cells and zebrafish embryos and that this stimulation is correlated with the binding of an inducible transcription factor, most probably the fish HSF, and a constitutive factor to this element.

We thank R. Carpeaux for excellent technical assistance, and R. Day for generously providing the pCMVsGFPLuc vector. This work was supported by the 'Région Wallone' (ULg 1815); the 'Services Fédéraux des Affaires Scientifiques, Techniques et Culturelles' (PAI P4/30 and 'Actions de Recherche Concertées' 95/00-193); the Fonds National de la Recherche Scientifique (FNRS) (3.4537.93 and 9.4569.95); and the EU (no. BI04-CT97-0554). M. M. is a Chercheur qualifié at the Fonds National de la Recherche Scientifique (FNRS). A. M. held fellowships from CGRI and 'ULg patrimoine'.

\section{REFERENCES}

1 Ritossa, F. (1962) A new puffing pattern induced by heat shock and DNP in Drosophila. Experientia 18, 571-573

2 Morimoto, R. I. (1998) Regulation of the heat shock transcriptional response: cross talk between a family of heat shock factors, molecular chaperones, and negative regulators. Genes Dev. 12, 3788-3796

3 Hartl, U. F. (1996) Molecular chaperones in cellular protein folding. Nature (London) 381, $571-580$

4 Holmgren, R., Corces, V., Morimoto, R., Blackman, R. and Meselson, R. (1981) Sequence homologies in the $5^{\prime}$ regions of four Drosophila heat shock genes. Proc. Natl. Acad. Sci. U.S.A. 78, 3775-3778

5 Pelham, H. R. (1982) A regulatory upstream promoter element in the Drosophila hsp70 heat shock gene. Cell 30, 517-528

6 Bienz, M. and Pelham, H. R. (1987) Mechanisms of heat shock gene activation in higher eukaryotes. Adv. Genet. 24, 31-72

7 Xiao, H. and Lis, J. T. (1988) Germline transformation used to define key features of heat shock response elements. Science 239, 1139-1142

8 Amin, J., Ananthan, J. and Voellmy, R. (1988) Key features of heat shock regulatory elements. Mol. Cell. Biol. 8, 3761-3769

9 Fernandes, M., Xiao, H. and Lis, J. T. (1994) Fine structure analyses of the Drosophila and Saccharomyces heat shock factor-heat shock element interactions. Nucleic Acids Res. 22, 167-173

10 Rabindran, S. K., Haroun, R. I., Clos, J., Wisniewski, J. and Wu, C. (1993) Regulation of heat shock factor trimer formation: role of a conserved leucine zipper. Science 259, 230-234

11 Morimoto, R. I. (1993) Cells in stress: transcriptional activation of heat shock genes. Science 259, 1409-1410

12 Baler, R., Dahl, G. and Voellmy, R. (1993) Activation of human heat shock genes is accompanied by oligomerization, modification, and rapid translocation of heat shock transcription factor HSF1. Mol. Cell. Biol. 13, 2486-2496

13 Sarge, K. D., Murphy, S. P. and Morimoto, R. I. (1993) Activation of heat shock gene transcription by heat shock factor 1 involves oligomerization, acquisition of DNAbinding activity, and nuclear localization and can occur in the absence of stress. Mol. Cell. Biol. 13, 1392-1407

14 Sistonen, L., Sarge, K. D. and Morimoto, R. I. (1994) Human heat shock factors 1 and 2 are differentially activated and can synergistically induce hsp70 gene transcription. Mol. Cell. Biol. 14, 2087-2099 
15 Nakai, A., Kawazoe, Y., Tanabe, M., Nagata, K. and Morimoto, R. I. (1995) The DNAbinding properties of two heat shock factors, HSF1 and HSF3, are induced in the avian erythroblast cell line HD6. Mol. Cell. Biol. 15, 5168-5178

16 Bevilacqua, A., Fiorenza, M. T. and Mangia, F. (1997) Developmental activation of an episomic hsp70 gene promoter in two-cell mouse embryos by transcription factor Sp1. Nucleic Acids Res. 25, 1333-1338

17 Bienz, M. and Pelham, H. R. (1986) Heat shock regulatory elements function as an inducible enhancer in the Xenopus hsp70 gene and when linked to a heterologous promoter. Cell 45, 753-760

18 Bevilacqua, A., Fiorenza, M. T. and Mangia, F. (2000) A developmentally regulated GAGA box-binding factor and Sp1 are required for transcription of the hsp70.1 gene at the onset of mouse zygotic genome activation. Development 127, 1541-1551

19 Kothary, R. K., Jones, D. and Candido, E. P. (1984) 70-Kilodalton heat shock polypeptides from rainbow trout: characterization of cDNA sequences. Mol. Cell. Biol. 4, 1785-1791

20 Arai, A., Naruse, K., Mitani, H. and Shima, A. (1995) Cloning and characterization of cDNAs for 70-kDa heat-shock proteins (Hsp70) from two fish species of the genus Oryzias. Jpn. J. Genet. 70, 423-433

21 Lele, Z., Engel, S. and Krone, P. H. (1997) hsp47 and hsp70 gene expression is differentially regulated in a stress- and tissue-specific manner in zebrafish embryos. Dev. Genet. 21, 123-133

22 Santacruz, H., Vriz, S. and Angelier, N. (1997) Molecular characterization of a heat shock cognate cDNA of zebrafish, hsc70, and developmental expression of the corresponding transcripts. Dev. Genet. 21, 223-233

23 Lim, E. H. and Brenner, S. (1999) Short-range linkage relationships, genomic organisation and sequence comparisons of a cluster of five HSP70 genes in Fugu rubripes. Cell Mol. Life Sci. 55, 668-678

24 Halloran, M. C., Sato-Maeda, M., Warren, J. T., Su, F., Lele, Z., Krone, P. H., Kuwada, J. Y. and Shoji, W. (2000) Laser-induced gene expression in specific cells of transgenic zebrafish. Development 127, 1953-1960

25 Molina, A., Biemar, F., Muller, F., Iyengar, A., Prunet, P., Maclean, N., Martial, J. A. and Muller, M. (2000) Cloning and expression analysis of an inducible HSP70 gene from tilapia fish. FEBS Lett. 474, 5-10

26 Poncelet, D. A., Bellefroid, E. J., Bastiaens, P. V., Demoitié, M. A., Marine, J. C., Pendeville, H., Alami, Y., Devos, N., Lecocq, P., Ogawa, T. et al. (1998) Functional analysis of the ZNF85 Krab zinc finger protein, a member of the highly homologous ZNF91 gene family. DNA Cell Biol. 17, 931-942

27 Poncelet, A. C., Levavi-Sivan, B., Muller, M., Yaron, Z., Martial, J. A. and Belayew, A (1996) The tilapia prolactin I gene: evolutionary conservation of the regulatory elements directing pituitary-specific expression. DNA Cell Biol. 15, 679-692

28 Day, R. N., Kawecki, M. and Berry, D. (1998) Dual-function reporter protein for analysis of gene expression in living cells. Biotechniques 25, 848-850, 852-854, 856

Received 16 January 2001/26 February 2001; accepted 28 March 2001
29 Fijan, N., Sulimanavoic, D., Bearzotti, M., Muzinic, M. D., Zwilenberg, L. 0 Chilmonzyk, S., Vautherot, J. F. and de Kinkelin, P. (1983) Some properties of the Epithelioma papulosum cyprini (EPC) cell line from carp Cyprinus carpio. Ann. Virol. 134, 207-220

30 Brasier, A. R., Tate, J. E. and Habener, J. F. (1989) Optimized use of the firefly luciferase assay as a reporter gene in mammalian cell lines. Biotechniques $\mathbf{7}$, 1116-1122

31 Sambrook, J., Fritsch, E. F. and Maniatis, T. (1989) Molecular Cloning: A Laboratory Manual, 2nd edn, Cold Spring Harbor Laboratory, Cold Spring Harbor, NY

32 Bradford, M. M. (1976) A rapid and sensitive method for the quantitation of microgram quantities of protein utilizing the principle of protein-dye binding. Anal. Biochem. 72, 248-254

33 Westerfield, M. (1993) The Zebrafish Book. A Guide for the Laboratory Use of Zebrafish (Danio rerio), University of Oregon Press, Eugene

34 Williams, G. T. and Morimoto, R. I. (1990) Maximal stress-induced transcription from the human HSP70 promoter requires interactions with the basal promoter elements independent of rotational alignment. Mol. Cell. Biol. 10, 3125-3136

35 Morimoto, R. I., Hunt, C., Huang, S. Y., Berg, K. L. and Banerji, S. S. (1986) Organization, nucleotide sequence, and transcription of the chicken HSP70 gene. J. Biol. Chem. 261, 12692-12699

36 Corces, V. and Pellicer, A. (1984) Identification of sequences involved in the transcriptional control of a Drosophila heat-shock gene. J. Biol. Chem. 259, 14812-14817

37 Gordon, S., Bharadwaj, S., Hnatov, A., Ali, A. and Ovsenek, N. (1997) Distinct stressinducible and developmentally regulated heat shock transcription factors in Xenopus oocytes. Dev. Biol. 181, 47-63

38 Yang, S. H., Nussenzweig, A., Yang, W. H., Kim, D. and Li, G. C. (1996) Cloning and characterization of rat Ku70: involvement of Ku autoantigen in the heat-shock response. Radiat. Res. 146, 603-611

39 Mosser, D. D., Theodorakis, N. G. and Morimoto, R. I. (1988) Coordinate changes in heat shock element-binding activity and HSP70 gene transcription rates in human cells. Mol. Cell. Biol. 8, 4736-4744

40 Nussenzweig, A., Sokol, K., Burgman, P., Li, L. and Li, G. C. (1997) Hypersensitivity of Ku80-deficient cell lines and mice to DNA damage: the effects of ionizing radiation on growth, survival, and development. Proc. Natl. Acad. Sci. U.S.A. 94, 13588-13593

41 Hsu, H. L., Gilley, D., Galande, S. A., Hande, M. P., Allen, B., Kim, S. H., Li, G. C., Campisi, J., Kohwi-Shigematsu, T. and Chen, D. J. (2000) Ku acts in a unique way at the mammalian telomere to prevent end joining. Genes Dev. 14, 2807-2812

42 Wilkins, R. C. and Lis, J. T. (1998) GAGA factor binding to DNA via a single trinucleotide sequence element. Nucleic Acids Res. 26, 2672-2678

43 Bevilacqua, A., Fiorenza, M. T. and Mangia, F. (2000) A developmentally regulated GAGA box-binding factor and Sp1 are required for transcription of the hsp70.1 gene at the onset of mouse zygotic genome activation. Development 127, 1541-1551 